\title{
Lupus-related myelopathy: report of three cases and review of the literature
}

\author{
James Provenzale, Thomas W Bouldin
}

\begin{abstract}
Transverse myelopathy is an uncommon complication of systemic lupus erythematosus (SLE). Three patients with SLE are reported who developed transverse myelopathy, including the neuropathological findings in one patient on whom necropsy was performed. Paraparesis was present in all three cases, but definite sensory changes were present in only one patient. In two patients, the CSF findings were remarkable for elevated protein and depressed glucose concentrations. Microscopic examination of the brain demonstrated small, scattered foci of recent necrosis consistent with microinfarctions. Striking abnormalities were found in the spinal cord at all levels, including multiple foci of vacuolar spongy degeneration in the peripheral white matter, as well as ballooning of myelin sheaths, swollen axons, myelin pallor, and loss of glial nuclei. The pathological findings in previously reported cases of SLE-related transverse myelopathy are reviewed, and the possible pathogenesis of the findings in our case are discussed.
\end{abstract}

(F Neurol Neurosurg Psychiatry 1992;55:830-835)

Although CNS complications of systemic lupus erythematosus (SLE) are common, ${ }^{1}$ descriptions of myelopathy related to SLE are relatively rare. Reported cases of lupus-related myelopathy have, for the most part, been notable for poor recovery and a high mortality rate. Three cases of myelopathy related to SLE have been treated at the North Carolina Memorial Hospital over a ten year period, which suggests that this condition is not as rare as the medical literature would indicate. We report these cases, including the pathological findings in one case.

\section{Case reports}

Case one

A 21 year old black woman developed intermittent fevers, night sweats, anorexia, a generalised rash and diffuse lymphadenopathy in February 1983. Initial serological studies for SLE were negative, and she was given a diagnosis of seronegative SLE. The rash responded to prednisone, $30 \mathrm{mg}$ four times a day. Subsequently she was found to have a hightitre speckled ANA and antibody to SS-A and RNP. She was treated with hydroxy- chloroquine for three months, and steroids were tapered to a low maintenance dose eight months after onset of symptoms.

Two years later, she developed fever, flank pain and malaise, and steroids were increased to $20 \mathrm{mg}$ daily. She also developed progressive gait difficulty over the next three weeks and experienced three generalised seizures. She had no urinary symptoms but bowel movements were decreased.

Neurological examination revealed moderate lethargy, poor short-term memory, normal cranial nerve examination, full strength in the arms without pronator drift but $2 / 5$ strength diffusely in both legs. Cerebellar and sensory examination were normal. She was treated with phenytoin and phenobarbital. CT head scan showed moderate cerebral atrophy. Myelographic examination of the entire spinal cord was normal. The lumbar puncture revealed clear, colourless fluid, with 0 RBC, 0 WBC, glucose $79 \mathrm{mg} \%$ (no simultaneous serum glucose), and protein $71 \mathrm{mg} \%$. The erythrocyte sedimentation rate was $130 \mathrm{~mm} /$ $\mathrm{hr}$, and the peripheral white blood cell count was $1000 / \mathrm{cu} \mathrm{mm}$. The initial diagnosis was lupus cerebritis and her prednisone was increased to $40 \mathrm{mg}$ daily. Over the next week, her paraparesis worsened, with absent deep tendon reflexes in the legs and extensor plantar responses, although strength in her arms was unaffected. She was treated with methylprednisolone $25 \mathrm{mg}$ twice a day.

One week later, she experienced a sudden hypotensive episode, and needed ventilatory support. She became increasingly disoriented. A repeat lumbar puncture showed clear, colourless fluid, $10 \mathrm{RBC}, 0 \mathrm{WBC}$, glucose $110 \mathrm{mg} \%$ (simultaneous serum glucose $236 \mathrm{mg} \%$ ) and protein $67 \mathrm{mg} \%$. Transverse myelitis secondary to SLE was considered the most likely diagnosis. She died after a respiratory arrest one week later, five weeks after onset of neurological symptoms.

At necropsy, gross examination of the brain revealed slight atherosclerotic changes of the arteries of the circle of Willis and no brain atrophy. The cranial nerves were unremarkable. A shaggy fibrinous exudate was present on the dura mater of the spinal cord. The gross appearance of the spinal cord was unremarkable. The cut surfaces of the brain and spinal cord showed no focal lesions. Microscopic examination of the brain revealed scattered microscopic foci of recent necrosis consistent with microinfarcts in the cerebral cortex, basal ganglia, thalamus and brainstem. The blood vessels in the effected areas of the brain often 
Figure 1 Anterior

funiculus of lumbar spinal cord from case 1 . Multiple areas of spongy

degeneration are present in the subpial white matter along the median fissure. Note normal blood vessels in the overlying

leptomeninges ( $\times 50$;

Solochrome-eosin stain).

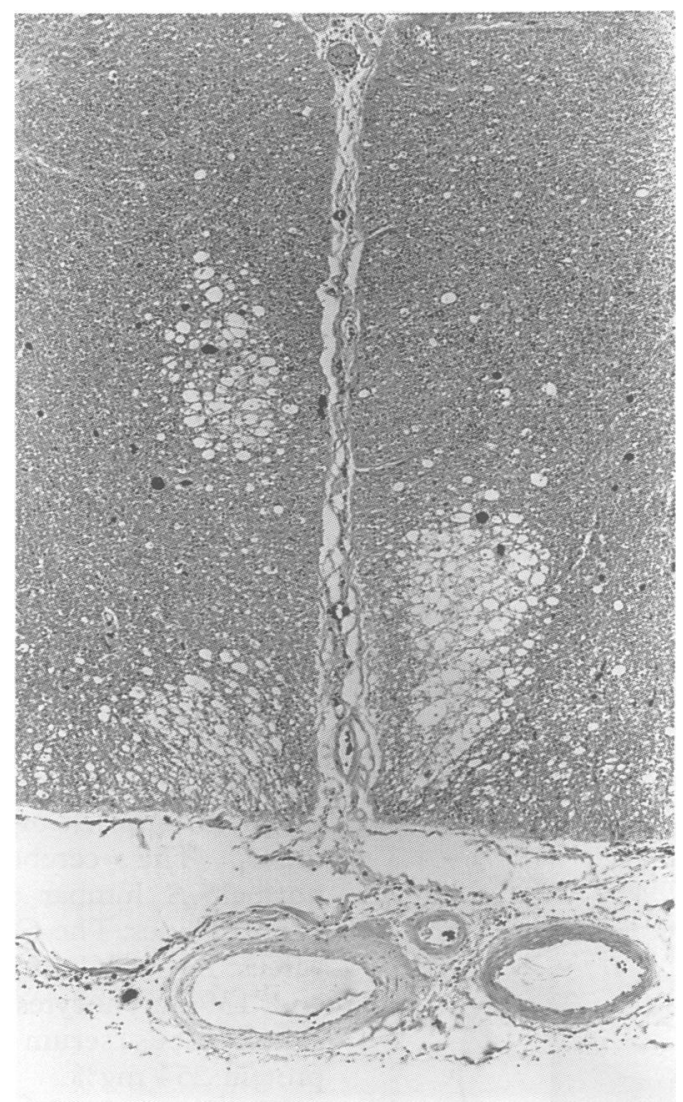

exhibited mural thickening with reactive endothelial cells. Occasional blood vessels had perivascular chronic inflammatory infiltrates, but neither necrotising arteritis, nor thrombi nor emboli were identified.

The most striking microscopic abnormalities in the spinal cord were multiple foci of spongy degeneration of the white matter in the periphery of the spinal cord (fig 1). These lesions were seen at all levels of the spinal cord but were most frequent at the lumbar level (fig 2). Similar spongy degeneration of the white matter was also focally present in the medulla. The lateral funiculus, especially the dorsolateral region, was involved at all levels. The posterior columns were relatively spared, while the anterior funiculus showed variable degrees of involvement, most severe at the lumbar level. There was no evidence of ascending or descending tract degeneration. The anterior horn cells and nerve roots were intact at all levels. The blood vessels surrounding the spinal cord did not reveal vasculitis, perivascular infiltrates, thrombi or emboli.

At higher magnification, the areas of spongy degeneration showed clear vacuoles and swollen axons (fig 3). Many vacuoles appeared to be surrounded by a thin rim of myelin, suggesting that these vacuoles represented ballooning of myelin sheaths. In some foci, occasional foamy macrophages were also present. The largest of the spongy lesions also showed pallor of myelin and pyknosis and loss of glial nuclei.

Case two

A 15 year old black female developed arthralgias, fatigue, fever and weight loss in 1984, and found to have an ESR of 148, low C3, C4 and $\mathrm{CH} 50$ titres, an ANA positive at 1:640 with a speckled pattern, and a positive rheumatoid factor at 1:640. Over the following year, she developed renal failure secondary to membraneous glomerulnephritis and pleural effusions despite treatment with prednisone $60 \mathrm{mg}$ daily. Her steroids were tapered over the next year to a low maintenance dose, but in late 1986 she developed confusion, dysarthria, slowly progressive leg weakness and pain, and urinary incontinence over the course of three weeks. At the time of admission to hospital three weeks after the onset of leg weakness, she was able to ambulate only with assistance. Short-term memory and calculations were poor. She had dysarthric speech but no other significant cranial nerve findings. Strength was rated $4 / 5$ in the arms, $2 / 5$ at the hips and $3 / 5$ at the knees. Deep tendon reflexes were hyperactive in the arms, with clonus at both knees and both ankles, and bilateral extensor plantar responses. Cerebellar and sensory examinations were normal. Her rectal sphincter tone was normal.

A lumbar puncture showed one red blood cell, seven white blood cells, glucose $41 \mathrm{mg} \%$ (no simultaneous serum glucose) and protein $248 \mathrm{mg} \%$. CSF gram stain showed no organisms, and CSF culture and AFB stain were negative. MRI of the brain and entire spinal cord revealed significant cerebral atrophy but no other abnormalities.

The patient was treated with $60 \mathrm{mg}$ daily of prednisone, but her confusion and leg weakness continued to worsen. She was given methylprednisolone $250 \mathrm{mg}$ intravenous four times a day for three days without improvement. Visual and brainstem auditory evoked potentials were normal. Upper extremity somatosensory evoked potentials were normal while lower extremity somatosensory evoked potentials were abnormal bilaterally. A repeat lumbar puncture revealed clear, colourless fluid, with 2 RBC, 1 WBC, glucose $65 \mathrm{mg} \%$ (simultaneous serum glucose $142 \mathrm{mg} \%$ ) and protein $41 \mathrm{mg} \%$. The CSF/serum IgG/albumin index was 2.91 (normal range $0.34-0.88$ ) and oligoclonal bands were noted in the CSF but not in the serum.

The patient had a series of 11 plasma exchange procedures over the course of the next four weeks, supplemented by an infusion of 0.75 grams of cyclophosphamide at one week. At the time of discharge three weeks after beginning plasma exchange therapy, significant improvement in the patient's encephalopathy was noted, with moderate improvement in leg strength and urinary bladder function. At the time of discharge she was able to walk with the aid of a walker.

The patient subsequently had another pulse infusion of 0.7 grams of cyclophosphamide and a single plasma exchange procedure one month after discharge. She was maintained on $60 \mathrm{mg}$ prednisone daily. Six months after the onset of leg weakness, she was able to walk without a walker, could climb stairs with assistance and her bladder function had returned to normal. 

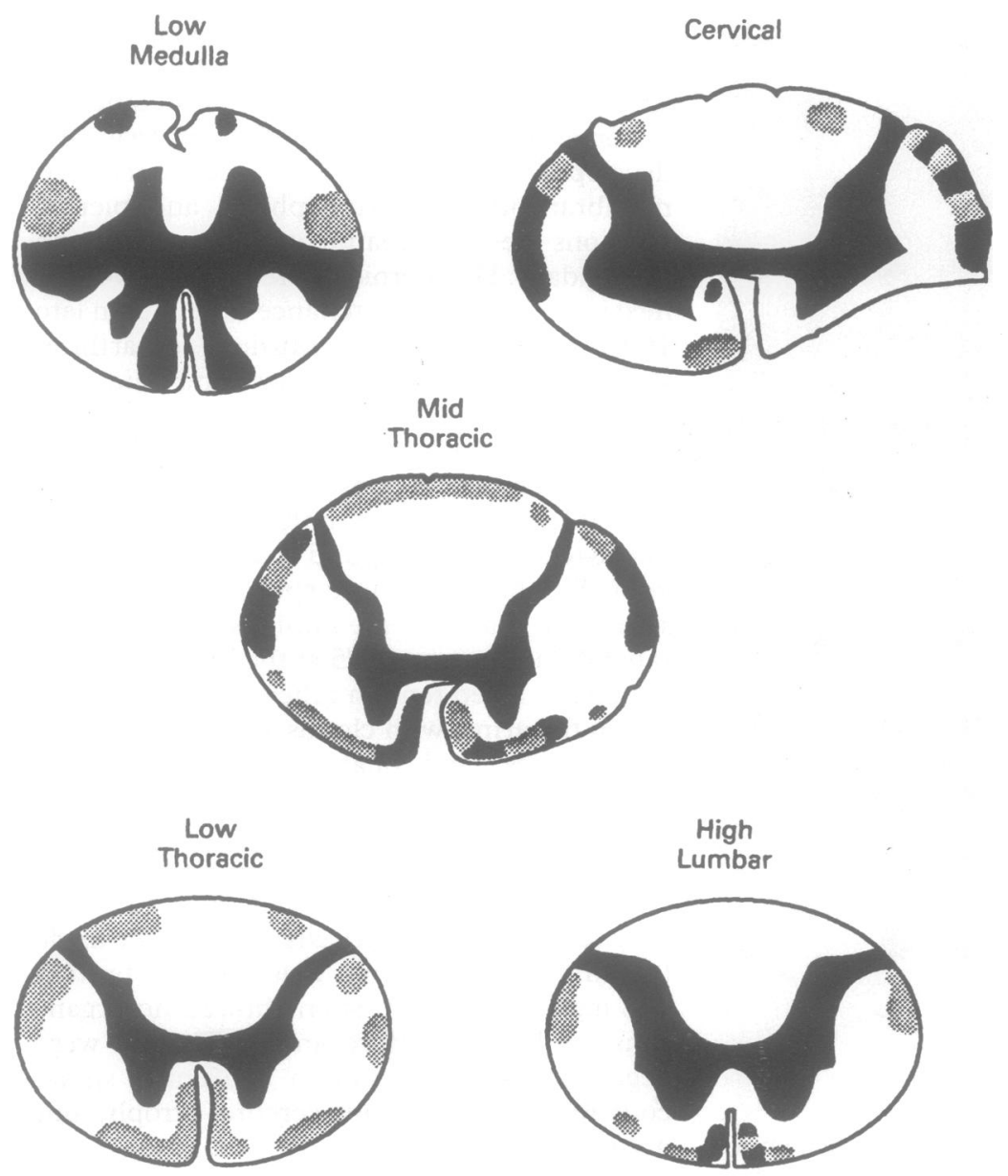

VENTRAL SURFACE

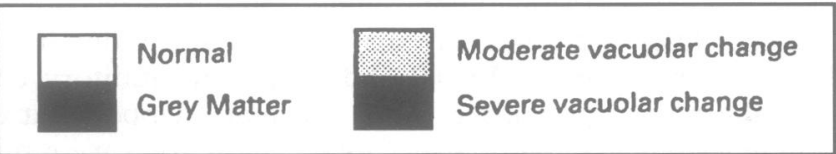

Figure 2 Schematic representation demonstrating the distribution of subpial vacuolar changes within the spinal cord white matter tracts at various levels.

\section{Case three}

A 15 year old black female developed a papular erythematous rash over her arms in 1977, and soon after experienced arthralgias, diffuse

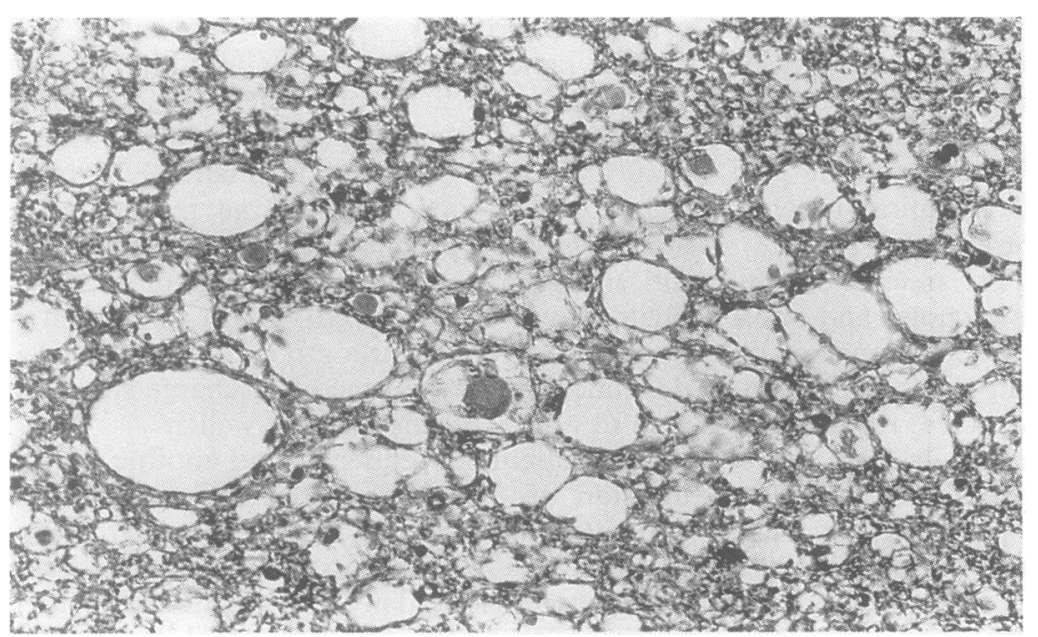

Figure 3 Region of white matter degeneration in anterior fasciculus of thoracic spinal cord exhibiting clear vacuoles rimmed by myelin. The dense, homogeneous structure near the centre of the field is a swollen axon. There is pyknosis and loss of glial nuclei, suggesting glial-cell necrosis and dropout ( $\times$ 380; Solochrome-eosin stain). weakness and alopecia. Laboratory studies showed a greatly elevated CPK as well as a positive LE prep of 1:2000, an ANA titre of $1: 320$, a negative rheumatoid factor and negative double-stranded DNA tests. An electromyogram showed polyphasia and reduced motor unit duration and a muscle biopsy was diagnostic of an inflammatory myopathy. She was diagnosed as having SLE and polymyositis. The patient was treated with $60 \mathrm{mg}$ prednisone daily with significant improvement and was tapered to low maintenance doses over the next year.

One year later, she developed low back pain, bilateral leg weakness and urinary incontinence which progressed over three weeks before admission to hospital. Her neurological examination revealed normal strength in the arms, but $3 / 5$ strength throughout the legs. She had normal reflexes in the arms but absent knee and ankle jerk reflexes, and bilateral extensor plantar responses. There was hypaesthesia to pinprick over the L3 to S5 dermatomes. The cerebellar examination was normal. A lumbar myelogram revealed no abnormalities. The CSF showed the following values: 206 RBC, 20 WBC (87\% lymphocytes and $13 \%$ monocytes), glucose $46 \mathrm{mg} \%$ (no simultaneous serum glucose obtained) and protein $254 \mathrm{mg} \%$.

The diagnosis of myelopathy related to SLE was made and her prednisone dosage was increased to $60 \mathrm{mg}$ daily. A repeat CSF examination a week later showed 52 red blood cells, 84 white blood cells ( $80 \%$ lymphocytes, $20 \%$ monocytes), glucose $105 \mathrm{mg} \%$ with simultaneous serum glucose of $139 \mathrm{mg} \%$, and protein of 261. The CSF/serum IgG/albumin ratio was 0.685 , and the fluid was positive for the presence of myelin basic protein. She improved slowly over the next two months and at discharge was able to walk with the aid of a walker, with significant resolution of sensory dysfunction, although a neurogenic bladder was still present. At six months after onset of neurological symptoms, she continued to show moderate leg weakness on low daily doses of prednisone, on which she is presently maintained.

\section{Discussion}

CNS complications of SLE have been reported to occur at some point in the majority of SLE cases. ${ }^{2}$ These include focal neurological findings such as cranial neuropathy, hemiparesis and hemisensory loss, as well as nonfocal changes such as psychosis, memory loss, disturbance of affect, and generalised seizures. However, lupus-related myelopathy, that is, quadriparesis or paraparesis, sensory changes in the extremities, and bowel and bladder dysfunction due to spinal cord involvement, has not been commonly reported. A review of the literature reveals a description of only 31 cases. Histopathological findings have been reported in less than half of these cases. Myelopathic features do not preclude the possibility of other CNS abnormalities, and in cases where seizures or disturbance of menta- 
tion accompany spinal cord-related symptoms and signs, the clinical syndrome is best described by the term "encephalomyelopathy".

The most characteristic presentation of lupus-related myelopathy in a patient with previously diagnosed SLE is the development of bilateral leg weakness and sensory changes with bowel and bladder dysfunction. These findings are almost invariably present. ${ }^{3}$ Signs referrable to a cervical spinal cord lesion, including bilateral arm weakness, have also been reported. ${ }^{3}$ However, paraparesis is much more commonly seen than quadraparesis. Occasionally neck or back pain of a rather significant degree is also present. ${ }^{4}$ Because these symptoms may mimic those of a spaceoccupying spinal cord lesion, such as an abscess, haematoma or neoplasm, myelography or MRI may be indicated in individual cases. $^{5}$

Extensor plantar responses have been found in 12 of 18 cases in which they have been recorded, including all three patients in our series. Sensory changes are described in almost all reported cases, with a mid- or low-thoracic sensory level being described most often. Incomplete sensory loss has been reported in a number of cases. In addition, occasionally sensory loss only in the sacral dermatomes has been reported, as in one of our cases. As illustrated in our first two cases, sensory loss need not be present. In the first case, inability to determine loss of all sensory modalities may be partly due to the difficulty in examining a severely encephalopathic patient. There is no singular characteristic change in the reflexes in the affected extremities. While some patients will have significantly diminished or absent reflexes, even in the setting of extensor plantar reflexes, some cases will have increased reflexes. As our cases illustrate, there may be a combination of diminished knee jerk reflexes and prominently increased ankle jerk reflexes.

The rate of onset is usually over the course of a few days to a week, although it is not uncommon for the neurological changes to develop over a few weeks. The interval from onset of SLE to the development of myelopathy varies from several months to a few years in most cases, although the presence of SLE symptoms up to 23 years before the onset of myelopathy has been reported. ${ }^{6}$ In rare instances, myelopathy has been reported as the first major manifestation of systemic lupus erythematosus, ${ }^{78}$ while in other cases there may have been other organ system involvement suggesting SLE without a definitive diagnosis.

The CSF formula is almost always abnormal at some point after the onset of myelopathy. The review by Warren and $\mathrm{Kredich}^{3}$ revealed elevated CSF protein levels in 14 of 17 patients, depressed CSF glucose levels (below $30 \mathrm{mg} \%$ ) in six of 12 patients, and a CSF pleocytosis of more than 20 cells $/ \mathrm{mm}$, usually lymphocytes, in 10 of 14 patients. The CSF protein levels were abnormally elevated (above $40 \mathrm{mg} \%$ ) in all of our patients at some point in their clinical course, but the degree of elevation differed significantly between cases and did not necessarily reflect the level of clinical involve- ment. In only one of our cases was the CSF glucose below $50 \mathrm{mg} \%$, although in case number one the CSF glucose was less than half of a simultaneous serum glucose level. Unfortunately, data regarding the serum glucose at the time of lumbar puncture are lacking in some of our cases and in most of the reported cases. Only one of our three cases showed a CSF pleocytosis of greater than 20 cells. CSF complement levels were not obtained in any of our cases but were reported to be depressed in two cases in the series of Warren and Kredich. ${ }^{8}$ In summary, CSF abnormalities are common in lupus-related myelopathy, but the abnormalities are nonspecific and often mild. Because of the nonspecific CSF abnormalities, and because these patients are often taking immunosuppressive drugs before the onset of myelopathy, bacterial, viral and fungal infections always need to be considered in the differential diagnosis.

Three types of pathological changes have been reported in lupus-related myelopathy (table). In the first, there is extensive spinal cord softening limited to a specific cord level, and accompanied by vascular alterations ranging from perivasculitis to fibrinoid arteritis to thrombosis. Sinkovics, ${ }^{9}$ Piper $^{10}$ and Adrianakos $^{11}$ have each reported cases in which there was extensive spinal cord infarction at a specific cord level.

The second type of pathological change associated with lupus-related myelopathy is a large subdural haematoma at a spnal cord level, unaccompanied by vasculopathy. There are two reported cases of myelopathy secondary to subdural haematoma and spinal cord compression in patients with SLE (table). In one case there were multiple small haemorrhages within the substance of the spinal cord, ${ }^{12}$ and in the other, there was necrosis of the underlying spinal cord parenchyma. ${ }^{13}$

The most common type of pathological change reported in cases of SLE-related myelopathy is peripheral white-matter degeneration, often at multiple spinal cord levels. We have termed this characteristic pattern of spinal cord degeneration "subpial leukomyelopathy" because of its peripheral location and its primary involvement of white matter. These lesions show ballooning of myelin sheaths and axonal degeneration similar to that seen in our first case. Spinal-cord tract degeneration is not seen in these cases. As opposed to the extensive spinal cord destruction at a specific cord level in the type I category, the extent of the spinal cord degeneration at any one level in subpial leukomyelopathy may appear relatively insignificant. The fact that the lesions result in the clinical presentation of paraparesis or quadriparesis and significant sensory abnormalities is apparently due to a summation effect.

Previously reported cases of subpial leukomyelopathy are outlined in the table. Vacuolar changes were reported at all spinal cord levels in a number of these cases, although in Fisher's patient the changes were primarily at the thoracic and lumbar levels. ${ }^{14}$ Andrews et al ${ }^{6}$ did not describe the characteristic peripheral distribution of vacuolar changes but their case 
Table Pathological findings in lupus myelopathy

\begin{tabular}{|c|c|c|}
\hline Author (citation) & Gross pathological findings & Microscopic findings \\
\hline \multicolumn{3}{|c|}{ Type I Large spinal cord infarct } \\
\hline Sinkovics 9 & $\begin{array}{l}\text { Spinal cord softening and loss of } \\
\text { cord substance at lumbar level. }\end{array}$ & $\begin{array}{l}\text { Infarction pattern with thrombosis of anterior spinal } \\
\text { artery. } \\
\text { Acute fibrinoid necrosis of wall of anterior spinal artery. } \\
\text { Cryptococcal meningeal infiltrate. }\end{array}$ \\
\hline Piper $^{10}$ & $\begin{array}{l}\text { Fusiform swelling of spinal cord at } \\
\text { lumbar level. }\end{array}$ & $\begin{array}{l}\text { Myelomalacia of posterior horns. Fibrinoid change and } \\
\text { perivascular infiltrate in adjacent meningeal vessels. } \\
\text { Thrombi in surrounding vessels. }\end{array}$ \\
\hline Adrianakos ${ }^{11}$ & $\begin{array}{l}\text { Large necrotic lesion throughout } \\
\text { middle thoracic cord segments. }\end{array}$ & $\begin{array}{l}\text { Necrosis of grey and white matter at thoracic spinal cord } \\
\text { level. Ascending and descending tract degeneration at } \\
\text { other spinal cord levels. } \\
\text { Perivascular connective tissue proliferation. Lymphocytic } \\
\text { and mononuclear cell infiltrates around small venules. } \\
\text { No evidence of occlusive lesion. }\end{array}$ \\
\hline \multicolumn{3}{|c|}{ Type II Spinal cord subdural hematoma } \\
\hline Johns Hopkins CPC ${ }^{12}$ & $\begin{array}{l}\text { Two large subdural haematomas } \\
\text { compressing lower spinal cord. }\end{array}$ & $\begin{array}{l}\text { Multiple small haemorrhages within substance of spinal } \\
\text { cord and dorsal root ganglia. No evidence of arteritis. }\end{array}$ \\
\hline Weil $^{13}$ & $\begin{array}{l}\text { Subdural haematoma extending } \\
\text { over low thoracic and upper } \\
\text { lumbar spinal cord. }\end{array}$ & Necrosis of underlying spinal cord. \\
\hline \multicolumn{3}{|c|}{ Type III Subpial leukomyelopathy } \\
\hline Andrews $^{6}$ & $\begin{array}{l}\text { Loss of spinal cord substance at } \\
\text { thoracic and lumbar spinal cord } \\
\text { levels. }\end{array}$ & $\begin{array}{l}\text { Areas of necrosis at all spinal cord levels, with } \\
\text { replacement by lipid-filled macrophages. No evidence } \\
\text { of vasculitis. }\end{array}$ \\
\hline Fisher $^{14}$ & $\begin{array}{l}\text { Loss of spinal cord substance at } \\
\text { lumbar levels. }\end{array}$ & $\begin{array}{l}\text { Ballooning of myelin sheaths in peripheral white matter at } \\
\text { thoracic and lumbar levels. Edema and neuronal } \\
\text { degeneration in grey matter. Haemorrhage in many } \\
\text { Virchow-Robin spaces. }\end{array}$ \\
\hline Gold and Yahr ${ }^{17}$ & Not described. & $\begin{array}{l}\text { Peripherally placed white matter degeneration involving } \\
\text { entire spinal cord circumference. }\end{array}$ \\
\hline Johnson and Richardson ${ }^{1}$ & Not described. & $\begin{array}{l}\text { Disruption of both myelin and axis cylinders. Vacuoles of } \\
\text { varying sizes showing swollen myelin sheaths at border } \\
\text { of normal and abnormal parenchyma. No evidence of } \\
\text { vasculitis. }\end{array}$ \\
\hline Penn and Rowan ${ }^{16}$ & $\begin{array}{l}\text { Multiple peripheral areas of } \\
\text { rarefaction of thoracic spinal } \\
\text { cord white matter. }\end{array}$ & $\begin{array}{l}\text { Loss of nerve fibres and occasional swollen and } \\
\text { degenerated axons. }\end{array}$ \\
\hline \multirow[t]{2}{*}{ Nakano $^{15}$} & $\begin{array}{l}\text { Case I: Multiple rounded lesions in } \\
\text { periphery of spinal cord at all } \\
\text { levels. }\end{array}$ & $\begin{array}{l}\text { Marked spongy changes and swollen axons. Focal areas of } \\
\text { necrosis, various stages of fibrinoid necrosis or } \\
\text { organised thrombus in peripheral vessels. }\end{array}$ \\
\hline & $\begin{array}{l}\text { Case II: Marked pallor of } \\
\text { peripheral areas in circumferen- } \\
\text { tial distribution. }\end{array}$ & $\begin{array}{l}\text { Prominent axonal loss and gliosis in spinal cord } \\
\text { periphery. Diffuse vascular changes in peripheral } \\
\text { arachnoid arteries including severe intimal thickening } \\
\text { and fibrinoid necrosis. }\end{array}$ \\
\hline
\end{tabular}

is included because, unlike those cases outlined under the Type 1 and Type 2 categories, there was spinal cord degeneration at multiple levels as well as the absence of vascular abnormalities. Nakano ${ }^{15}$ described areas of marked axonal degeneration in the periphery of the spinal cord white matter in two patients with SLE-related transverse myelopathy, including focal regions of spongy change and swollen axons similar to the findings in our necropsy case. However, Nakano also reported vascular changes, including fibrinoid necrosis and severe intimal thickening and foamy cell infiltration, in the small arachnoid arteries of the spinal cords of both patients. Examination of the spinal cord vasculature in our case did not reveal similar changes.

It is not clear whether the three types of pathological changes in the spinal cord in lupus-related myelopathy are due to separate or common pathogenetic mechanisms. The primary abnormality in the first type of spinal cord degeneration, that is, a large infarct, seems to be due to thrombosis superimposed upon necrotising arteritis. Whether a primary defect of the vessel wall, such as some degree of fibrinoid necrosis leading to vascular weakening, is also present in the cases of spinal cord subdural haematoma is open to speculation. However, fibrinoid necrosis of the vessel wall was not mentioned in either of the two reported cases of spinal cord subdural haematoma. Penn and Rowan ${ }^{16}$ speculated that subdural haemorrhage in these cases may be secondary to a coagulopathy, for example, due to circulating lupus anticoagulant, in which case a primary mural abnormality need not be implicated.

The pathogenesis of the subpial leukomyelopathy in SLE is also uncertain. Gold and $\mathrm{Yahr}^{17}$ suggested that the pathological changes reported in their case were due to an autoimmune mechanism causing direct parenchymal damage. A circulating factor in the CSF might account for the peripheral distribution of the vacuolar changes seen in these cases, but such a factor has never been identified.

Similar vacuolar changes have been described in AIDS-related myelopathy. Petito $^{18}$ described these changes in $29 \%$ of consecutive necropsy cases of AIDS. The vacuolar degeneration was more prominent in patients with severe myelopathy. The changes were reported to be distributed throughout the spinal cord white matter, but were more prominent in the lateral columns. Like the changes seen in SLE, these abnormalities were not confined to specific anatomical tracts. As in the SLE cases, the vacuoles were surrounded 
by a thinned myelin sheath, suggesting that they were formed by intramyelin swelling. Similar findings have subsequently been described by other investigators. ${ }^{19}$ None of the patients in our series were known to have been exposed to any of the risk factors for AIDS, and neither of the two surviving patients has subsequently developed AIDS-like symptoms.

The peripheral distribution of the white matter degeneration in the spinal cord is similar to that described by Bunge and Settlage $^{20}$ following CSF barbotage in cats. A similar peripheral pattern of degeneration in spinal cord, brainstem and optic tracts was reported by Friede and Roessman ${ }^{21}$ in a patient who had repeated lumbar punctures for a CSF leak following transsphenoidal hypohysectomy. Winkelman ${ }^{22}$ also reported peripheral spongy degeneration of the spinal cord following accidental intrathecal injection of detergent solutions during administration of spinal anaesthesia.

The possibility that these lesions are secondary to ischaemia must also be considered. Nakano $^{15}$ reported fibrinoid necrosis and severe intimal thickening in arterioles surrounding the spinal leukomyelopathy. Similar vascular changes were not found in our case or the other previously reported cases and the subpial location of the changes is not the typical location of spinal cord infarcts. Thus there is little support for the hypothesis that these lesions are related to ischaemia.

In summary, lupus myelopathy may present with a variety of signs and symptoms, which may initially lead to misdiagnosis. Although paraparesis appears to be a constant feature, there is great variability regarding sensory disturbance and changes in the reflexes. There is also great variability in the CSF findings. Pathological findings similar to those seen in our first case have been previously described, and may be characteristic of lupus myelitis, although not pathognomonic of it. Unfortunately, at present, the pathogenesis of these findings is poorly understood.
1 Johnson RT, Richardson EP. Neurological manifestations of systemic lupus erythematosus. A clinicopathological study of twenty cases and review of the literature. Medicine (Baltimore) 1968;47:337-69.

2 Hughes GRV. Central nervous system lupus-diagnosis and treatment. F Rheumatol 1980;7:405-11.

3 Warren RW, Kredich BW. Transverse myelitis and acute central nervous system manifestations of systemic lupus central nervous system manifestations of systemic lupus
erythematosus. Arthritis and Rheumatism 1984;27: erythematos

4 Kewalramani LS, Saleem S, Bertrand D. Myelopathy Associated with Systemic Lupus Erythematosus. Paraplegia 1978-79;16:282-94.

5 Henry AK, Brunner CM. Relapse of lupus transverse myelitis mimicked by vertebral fractures and spinal cord compression. Arthritis and Rheumatism 1985;28: 1307-11.

6 Andrews JM, Cancilla PA, Kunin J. Progressive spinal cord signs in a patient with disseminated lupus erythematosus. Bull LA Neurologic Association 1970;35:78-85.

7 Siekert RG, Clark EC. Neurologic signs and symptoms as early manifestations of systemic lupus erythematosus. Neurology 1955;5:84-8.

8 Granger DP. Transverse myelitis with recovery: the only manifestation of systemic lupus erythematosus. Neurology 1960;10:325-9.

9 Sinkovics JG, Gyorkey F, Thoma GW. A rapidly fatal case of systemic lupus erythematosus: structure resembling viral nucleoprotein strands in the kidney and activities of lymphocytes in culture. Texas Reports Biol Med 1969;27: 887-908.

10 Piper PG. Disseminated lupus erythematosus with involvement of the spinal cord. $\mathcal{F} A M A 1953 ; 153: 215-7$.

11 Adrianakos AA, Duffy J, Suzuki M, Sharp JT. Transverse myelitis in systemic lupus erythematosus: report of three cases and review of the literature. Ann Int Med 1975; 83:616-24.

12 Clinicopathologic Conference. Bull fohns Hopkins Hospital 1966;118:423-37.

13 Weil MH. Disseminated lupus erythematosus with massive hemorrhagic manifestations and paraplegia. Lancet 1955; hemorrhagic

14 Fisher JH, Gilmour JR. Encephalomyelitis following administration of sulphanilimide. Lancet 1939;2:301-5.

15 Nakano I, Mannen T, Mizutani T, Yokohari R. Peripheral white matter lesions of the spinal cord with changes in small arachnoid arteries in systemic lupus erythematosus. Clin Neuropathol 1989;8:102-8.

16 Penn AS, Rowan AJ. Myelopathy in systemic lupus erythematosus. Arch Neurol 1968;18:337-49.

17 Gold AP, Yahr MD. Childhood lupus erythematosus. A clinical and pathological study of the neurological manifestations. Trans Am Neurolog Assoc 1960;85:96-102.

18 Petito CK, Navia BA, Cho ES, et al. Myelopathy pathologically resembling subacute combined degeneration in ologically resembling subacute combined degeneration in
patients with the acquired immunodeficiency syndrome. patients with the acquired

19 Rhodes RH. Histopathology of the central nervous system in the acquired immunodeficiency syndrome. Human Pathol 1987;18:636-43.

20 Bunge RP, Settlage PH. Neurological lesions in cats following cerebrospinal fluid manipulation. $\mathcal{F}$ Neuropath Exp Neurol 1957;16:471-91.

21 Friede RL, Roessman U. Destruction of peripheral white matter of the spinal cord, brainstem and optic tracts. f Neurol Neurosurg Psychiatry 1969;32:38-42.

22 Winkelman NW. Neurologic symptoms following accidental intraspinal detergent injection. Neurology 1952;2: 284-91. 